\title{
Non-gaussian velocity distribution for the LMC sources: microlensing implications
}

\author{
Luigi Mancini ${ }^{* \dagger}$ \\ Dipartimento di Fisica “E.R. Caianiello”, Università di Salerno, via S. Allende, Baronissi (SA), \\ Italy \\ E-mail: lmancini@physics.unisa.it
}

\begin{abstract}
We discuss whether the Gaussian is a reasonable approximation for the velocity distribution of stellar systems that are not spherically distributed. In particular, by using a non-Gaussian velocity distribution to describe the sources in the Large Magellanic Cloud (LMC), we reinvestigate the expected microlensing parameters of a lens population isotropically distributed either in the Milky Way halo or in the LMC (self lensing) and compare our estimates with the experimental results of the MACHO collaboration. An interesting result that emerges from our analysis is that, moving from the Gaussian to the non-Gaussian case, we do not observe any change in the form of the distribution curves of the rate of microlening events for lenses in the Galactic halo. Moreover, the corresponding expected timescales and the number of the expected events do not vary. Conversely, with regard to the self-lensing case, we observe a moderate increase of the rate and the number of expected events. The main conclusion is that the error on the estimate of the most likely value for the MACHO mass as well as for the Galactic halo fraction in form of MACHOs, calculated with a Gaussian velocity distribution for the LMC sources, is not grater than $2 \%$.
\end{abstract}

The Manchester Microlensing Conference: The 12th International Conference and ANGLES Microlensing Workshop

January 21-25 2008

Manchester, $U K$

\footnotetext{
* Speaker.

${ }^{\dagger}$ The author acknowledge support for this work by funds of the Regione Campania, L.R. n.5/2002.
} 


\section{Introduction}

Today many aspects of the features of the galaxies are still unclear. In particular, it is not obvious how describe the velocities of the constituents (in particular stars) of their components, just because we can not consider them isotropically distributed at any point. Actually, little is known about the velocity distribution (VD) of the stellar populations of galactic components. While the distribution of stellar velocities in an elliptical galaxy is generally reasonably close to a Gaussian, analyses of the line-of-sight velocity distributions of disk galaxies have shown that these distribution are highly non-Gaussian [1].

One of the most important problem regarding the composition of the Milky Way (MW) concerns the existence of dark compact agglomerates of baryons in the Galactic halo, the so called MACHOs (MAssive Compact Halo Objects). From the experimental point of view, in the course of more than ten years, several observational groups tried to detect these objects by performing microlensing surveys essentially in the directions of the Large Magellanic Cloud (LMC), Small Magellanic Cloud and M31. Two groups (MACHO and POINT-AGAPE) reported similar conclusions, despite the fact that they observed different targets (LMC and M31), that is roughly $20 \%$ of the Halo mass must be in form of MACHOs [2, 3], and the most likely MACHO mass is $\simeq 0.5$ $\mathbf{M}_{\odot}$. However, the interpretation of their data is controversial because of the insufficient number of events detected, and the existing degeneration among the parameters. Discordant results have been reported by other experimental teams $[4,5]$. Here we reexamine the framework of microlensing towards the LMC, and in particular we recalculate the number of expected events supposing that the source velocities are no longer Gaussian distributed. Both the MACHO and the self-lensing cases have been considered. We recalculate also the probability isocontours in the $m, f$ plane, where $m$ is the most likely MACHO mass, and $f$ is the Galactic halo fraction in form of MACHOs.

\section{Non-Gaussian velocity distributions}

If we consider a spherically symmetric distribution of stars with density $\rho$, then we can describe the dynamical state of the system by a distribution function of the following form

$$
F(E)=\frac{\rho}{\left(2 \pi \sigma^{2}\right)^{3 / 2}} e^{E / \sigma^{2}},
$$

where $E=\Psi-v^{2} / 2$ is the binding energy per unit mass, and $\Psi$ is the relative gravitational potential [6]. It is well known that the structure of a collisionless system of stars, whose density on phase space is given by Eq. (2.1), is identical to the structure of an isothermal self-gravitating sphere of gas. Therefore the velocity distribution at each point in the stellar-dynamical isothermal sphere is just the Maxwellian distribution $f(v)=N \exp \left(-\frac{1}{2} v^{2} / \sigma^{2}\right)$ exactly equal to the equilibrium Maxwell-Boltzmann distribution given by the kinetic theory.

Now, if we consider a stellar system that is very far from having a spherical distribution (for example a galactic flattened disk, a triaxial bulge, or an elongated bar), we do not expect that it is correct to use a Maxwellian distribution to describe its velocity profile. In the same way, we must ask if it is correct or not to use a Gaussian shape $f(v) \sim \exp \left(-\left(v^{2} / \sigma^{2}\right)\right)$ to describe the line-ofsight or the projected velocity profiles of non-spheroidal galactic components. We are going to 
answer this question in $\S 3$ and $\S 4$. We now introduce two non-Gaussian VDs that we will utilize to achieve the objective.

\subsection{Superposition of Gaussian distributions}

N-body simulations of different processes of galaxy formation have been performed by Iguchi et al. [7]. As a result of their simulations, these authors found stationary states characterized by a velocity distribution that is well described by an equally weighed superposition of Gaussian distributions of various temperatures, a so-called democratic temperature distribution (DT distribution), that is

$$
f_{\mathrm{DT}}(v)=\frac{1}{\sigma^{2}}\left\{\sqrt{\frac{2}{\pi}} \sigma e^{-v^{2} /\left(2 \sigma^{2}\right)}-|v|\left[1-\operatorname{Erf}\left(\frac{|v|}{\sqrt{2} \sigma}\right)\right]\right\},
$$

where $\operatorname{Erf}(x)$ is the error function. The conclusion was that the DT velocity distribution is a universal property of self-gravitating structures that undergo violent gravitational mixing. The origin of such universality remains however unclear.

\subsection{Universal velocity distribution}

Hansen et al. [8] have performed a set of simulations of controlled collision experiments of separately purely collisionless systems formed by self-gravitating particles. They have considered structures initially isotropic as well as highly anisotropic. After a strong perturbation followed by a relaxation, the final structures were not at all spherical or isotropic. Then, the VD extracted from the results of the simulations has been split into the radial and tangential parts. In this way they have found that the radial and tangential VDs are universal since they depend only on the radial or tangential dispersion and the local slope of the density; the density slope $\alpha$ is defined as the radial derivative of the density $\alpha \equiv(\mathrm{d} \ln \rho) /(\mathrm{d} \ln r)$. The points obtained by the simulations, which describe the universal tangential VD, are well fitted by the following functional form

$$
f_{\tan }\left(v_{\tan }\right)=\frac{v_{\tan }}{k^{2} \pi \sigma_{\tan }^{2}}\left(1-(1-q)\left(\frac{v_{\tan }}{k \sigma_{\tan }}\right)^{2}\right)^{q /(1-q)}
$$

where $\sigma_{\tan }$ is the tangential velocity dispersion, $v_{\tan }$ is the two-dimensional velocity component projected on the plane tangential to the 1.o.s., while $q$ and $k$ are free parameters. Hansen et al. [8] reported the universal tangential VD for three different values of the density slope $\alpha$. Here we will use the intermediate case with $\alpha$ equal to -2 . This VD has a characteristic break, with $v_{\tan }=$ $1.6 \sigma_{\tan }$ taken as the transition velocity. The low energy part is described by $q=5 / 3$ and $k=0.93$. Instead, for the high energy tails, the parameters are $q=0.82$ and $k=1.3$ (Hansen 2007, private communication).

\section{Microlensing towards the LMC revisited}

\subsection{State of the art}

While the MW is a well formed spiral galaxy, the LMC is an irregular galaxy, which presents two main components: a disk and a central bar. Moreover, the LMC is tilted with respect to the 
plane of the sky, with the north-east side closer to us than the south-west. In recent years different observational campaigns towards the LMC (MACHO, EROS, OGLE, MOA, SUPERMACHO) have been performed or are still working with the aim to detect MACHOs. Among these, only the MACHO and EROS groups have published their results. The EROS collaboration, started to take data in 1991, improved its experiment in 1996 becoming EROS 2 and finished the data taking in February 2003. They detected no events [4]. Instead, the MACHO Project, finished in 1999 after 5.7 years of continuous monitoring, detected 16 microlensing events, and concluded that MACHOs are a substantial constituent of the Galactic halo, but not the dominant component [2]. The corrected final estimate of the optical depth was $\tau=1.0 \pm 0.3 \times 10^{-7}$, whereas the maximum likelihood estimate of the mass of the lensing objects was $\approx 0.5 \mathrm{M}_{\odot}[9,2]$. Finally, the fraction $f$ of dark matter in form of MACHOs in the Galactic halo was estimated to be $\sim 20 \%$ [2]. Yet, the interpretation of these data is still a matter of controversy.

\subsection{Microlensing rate and number of expected events}

In the numerical estimates of the microlensing parameters, useful to study the fraction of the Galactic halo in form of MACHOs, a Gaussian shape velocity distribution is still commonly used to describe the projected velocity distribution for the lenses as well as the source stars, although they are not spherically distributed $[10,11,12,13]$. Here, our intention is to utilize the non-Gaussian velocity profiles discussed in the previous section for the sources, in place of the usual gaussian shape, and show how the microlensing probabilities change accordingly.

As a concrete case, we have analyzed two main parameters of the microlensing towards the LMC, the rate and the number of expected microlensig events generated by a lens population belonging to the MW halo as well as one belonging to the LMC itself. The results of our model have been compared with the MACHO collaboration observational results [2]. Actually, we restrict our analysis by considering a homogeneous subset of 12 Paczyński-like events taken from the original larger set B reported by MACHO [2]; we do not consider the Galactic disk events MACHO-LMC5 [14] and MACHO-LMC-20 [15], the binary event MACHO-LMC-9 [16], as well as all those candidates whose microlensing origin has been put in doubt, that is MACHO-LMC-22 [2] and MACHO-LMC-23 [9].

In our calculations we use the models presented in [11] to represent the various galactic components: essentially an isothermal sphere for the Galactic halo, a sech ${ }^{2}$ profile for the LMC disk and a triaxial boxy-shape for the LMC bar. The values of the parameters are the same as those of [11], except for the distance of the Sun from the Galactic center and the core radius of the Galactic halo, here fixed to 8 and $5 \mathrm{kpc}$ respectively.

A recent measure of the velocity dispersion of the LMC source stars gives $20.2 \mathrm{~km} / \mathrm{s}$ [17]. This measure is made as usual by a quantitative analysis of the absorption lines in the LMC spectrum, through a Gaussian form for the VD. In principle, in order to obtain an estimate of the velocity dispersion for a non-Gaussian distribution, we have to repeat the same analysis of the LMC line profile by applying now a non-Gaussian algorithm. In a first approximation, we will ignore this subtlety and simply impose the equality of the dispersion between the Gaussian and non-Gaussian VD.

In general the velocity of the lenses $v_{\ell}$ consists of a global rotation plus a dispersive component. Since we suppose that the MW halo has a spherical form, we can consider that the lenses 
are spherically distributed. In this case, the rotational component can be neglected, and at the same time we can safely consider the distribution of the dispersive component to be isotropic and Maxwellian [18].

It is well-known that the number of events $N$ is just the sum, $N=\sum N_{\text {field }}$, of the number of events expected for each monitored field of the experiment defined as

$$
N_{\text {field }}=E_{\text {field }} \int_{0}^{\infty} \frac{\mathrm{d} \Gamma}{\mathrm{d} T_{\mathrm{E}}} \mathscr{E}\left(T_{\mathrm{E}}\right) \mathrm{d} T_{\mathrm{E}}
$$

where $E_{\text {field }}$ is the field exposure, $\mathrm{d} \Gamma / \mathrm{d} T_{\mathrm{E}}$ is the differential rate with respect to the observed event duration, $T_{\mathrm{E}}$ is the Einstein time. $\mathscr{E}\left(T_{\mathrm{E}}\right)$ is the detection efficiency of the experiment [2]. The differential rate is defined as $[11,13])$

$$
\begin{aligned}
\frac{\mathrm{d} \Gamma}{\mathrm{d} T_{\mathrm{E}}} & =\int_{0}^{2 \pi} \mathrm{d} \alpha \int_{0}^{2 \pi} \mathrm{d} \varphi \int_{-\pi / 2}^{\pi / 2} \cos \theta \mathrm{d} \theta \int_{0}^{\infty} f\left(v_{\mathrm{s}}\right) \mathrm{d} v_{\mathrm{s}} \int_{\mu_{\min }}^{\mu_{\max }} \frac{R_{\mathrm{E}}}{\mathscr{N} n(x)} \frac{\mathrm{d} \mu}{\mathrm{d} \mu \times} \\
& \times \int_{0}^{\infty} \frac{v_{\ell}^{2}}{2 \pi \sigma_{\ell}^{2}} \exp \left(-\frac{v_{\ell}^{2}+x^{2} v_{\mathrm{s}}^{2}+2 x v_{\ell} v_{\mathrm{s}} \cos \varphi}{2 \sigma_{\ell}}\right) \mathrm{d} v_{\ell} \int_{0}^{1} \rho_{\ell}(x) x \mathrm{~d} x \int_{d_{\min }}^{d_{\min }} \rho_{\mathrm{s}}\left(D_{\mathrm{os}}\right) D_{\mathrm{os}} \mathrm{d} D_{\mathrm{os}}
\end{aligned}
$$

where $\rho_{\ell}$ and $\rho_{\mathrm{s}}$ are the lens and the source density respectively, $f\left(v_{\mathrm{s}}\right)$ represents the two-dimensional transverse velocity distribution of the sources, $x$ is the ratio between the observer-lens distance $D_{\mathrm{ol}}$ and the observer-source distance $D_{\mathrm{os}}$, whereas $\mu$ is the lens mass in solar mass units. The normalization factor $\mathscr{N}$ is the integral over the line of sight of the sources. $R_{\mathrm{E}}$ is the Einstein radius. The distribution $\mathrm{d} n(x) / \mathrm{d} \mu$ represents the number of lenses with mass between $\mu$ and $\mu+\mathrm{d} \mu$ at a given point in the Galactic halo. Assuming the factorization hypothesis, we can write $\mathrm{d} n(x) / \mathrm{d} \mu$ as the product of a distribution $\mathrm{d} n_{0} / \mathrm{d} \mu$ depending only on $\mu$ and the pertinent density profile [18]. For the functional form of $\mathrm{d} n_{0} / \mathrm{d} \mu$, we suppose that the mass function is peaked at a particular mass $\mu_{0}$, so that it can be described by a delta function. Concerning the functional form of $\mathrm{d} n_{0} / \mathrm{d} \mu$, we suppose that for the lenses in the Halo the mass function is peaked at a particular mass $\mu_{0}$, so that it can be described by a delta function. Instead, for lenses in the LMC disk/bar, we utilize an exponential form ([19])

\subsubsection{Lenses in the Galactic halo}

By using Eq. (3.3), we have calculated the differential rate of the microlensing events with respect to the Einstein time, along the lines pointing towards the events found by the MACHO collaboration in LMC and for different values of $\mu_{0}$. We have used a Gaussian VD for $f\left(v_{\mathrm{s}}\right)$ as well as the non-Gaussian VDs, Eq. (2.2) and Eq. (2.3). As $\mu_{0}$ and the l.o.s. change, we do not observe any substantial reduction in the height of the distribution curve of microlening event rate and the corresponding expected timescale does not vary among the cases considered.

With regard to the number of events, the situation does not change. Taking into account the MACHO detection efficiency and the total exposure $\left(6.12 \times 10^{7}\right.$ object-years $)$, we have calculated the expected number of events, summed up over all the fields examined by the MACHO collaboration in the case of a Halo completely (100\%) made of MACHOs. Both in the Gaussian and the non-Gaussian case, we have achieved the well-known result that the expected number of events is roughly 5 times larger than that observed. 


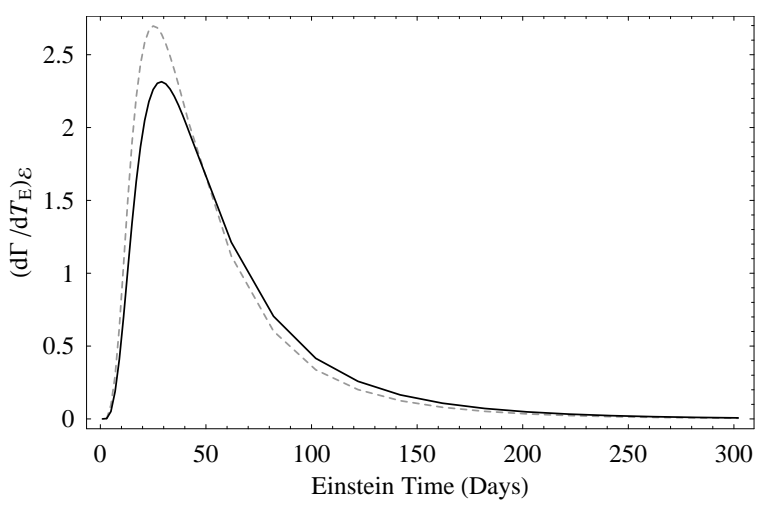

Figure 1: Self-lensing case. (a) Microlensing differential rate versus the Einstein time along the line pointing towards the event MACHO-LMC- 8 for $\mu_{0}=0.5 \mathrm{M}_{\odot}$; the $\mathrm{y}$-axis values are in $10^{-12}$ units. (b) Total number of microlensing events expected as a function of the lens mass. In the graphics two different curves are shown according to the velocity profile adopted for the sources: a Gaussian shape (solid line) and a universal VD (gray dotted line).

\subsubsection{Self lensing}

We repeat the same analysis also for the self-lensing configuration, that is both the lenses and the sources are located in the disk/bar of the LMC. In Figure 1 we report the differential rate versus the Einstein time $T_{\mathrm{E}}$ along the line pointing towards the event MACHO-LMC-8. The solid curve represents the Gaussian case, whereas the dashed curve has been obtained by using the universal VD (the results are similar if we use the DT distribution) for the LMC sources. In general, varying the l.o.s, we find that the microlensing differential rate for the non-Gaussian case is greater than that of the Gaussian case. We notice that the expected timescale varies too. In fact, moving from the Gaussian to the non-Gaussian case, we observe that the median value of the asymmetric distributions decreases (in Figure 1 it reduces from 51 to 46 days).

Concerning the expected number of microlensing events, fixing all the parameters according to the MACHO experiment, we have estimated that for sources having a non-Gaussian VD the number of expected events increases by roughly $35 \%$ from the value of 1.2 events obtained with a Gaussian VD (Mancini et al. [11]).

\subsection{MACHO Halo fraction and mass}

Following the methodology used in [2], namely the method of maximum likelihood, we estimate the halo fraction $f$ in form of MACHOs and the most likely MACHO mass. The likelihood function is

$$
L(m, f)=\exp \left(-N_{\mathrm{exp}}\right) \prod_{i=1}^{N_{\mathrm{obs}}}\left[E \mathscr{E}\left(T_{\mathrm{E}_{i}}\right) \frac{\mathrm{d} \Gamma}{\mathrm{d} T_{\mathrm{E}}}\left(T_{\mathrm{E}_{i}}, m\right)\right],
$$

where $N_{\text {exp }}$ is the total number of expected events, while the differential rate $\mathrm{d} \Gamma / \mathrm{d} T_{\mathrm{E}}$ is the sum of the differential rates of the lens populations (MACHOs, LMC halo, LMC disk+bar). The MACHO contribution is multiplied by $f$. The product runs over the $N_{\text {obs }}$ observed events. The resulting likelihood contours are shown in Fig. 2, where the estimate of the differential rate has been performed using a universal VD with $\alpha=-2$ to describe the motion of the LMC sources. The probabilities 


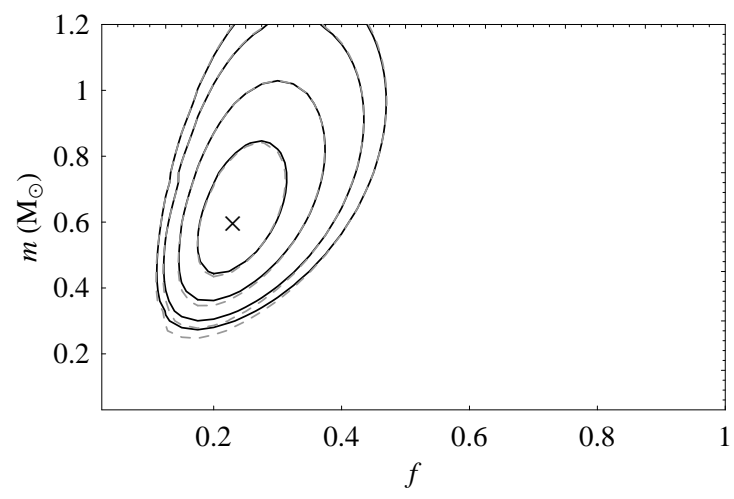

Figure 2: Likelihood contours for MACHO mass $m$ and Halo fraction $f$ for a typical spherical Halo. The contours have been obtained by using a universal VD for the LMC sources and enclose region of 34\%, 68\%, $90 \%$, and $95 \%$ probability. The cross shows the maximum-likelihood estimate.

are computed using a Bayesian method with a prior uniform in $f$ and $m$. A spherical isothermal distribution has been used to describe the lens density in the MW and LMC haloes. We find that the most probable mass is $m_{\mathrm{ML}}=0.69_{-0.22}^{+0.27} \mathrm{M}_{\odot}$, where the errors are $68 \%$ confidence intervals, and $f_{\mathrm{ML}}=27 \%$ with a $95 \%$ confidence interval of $13 \%-51 \%$. Here the subscript "ML" indicates maximum likelihood. We note that these values are slightly higher, though fully compatible, than the original result reported in [2]. The difference is due to some difference in the modelling and in the fact that the set of the events considered is smaller.

If we consider as usual that the velocities of the stars in the LMC are Gaussian distributed, the likelihood contours have very minimal differences from those of Fig. 2, in agreement with the results for the rate shown in $\S 3.2 .1$.

\section{Discussion and conclusion}

In order to describe as correctly as possible the motion of a stellar population having a nonspheroidal distribution we utilize two velocity distributions extracted by numerical simulations of collionsless systems formed by self-gravitating particles. These VDs are substantially different from a Gaussian one. As a concrete case, we consider the stars in the disk and bar components of the LMC and investigate on their potential power to be sources of lensing by transient MACHOs. In this framework we have recalculated the main microlensing parameters as well as the MACHO Halo fraction and the most likely value for the lens mass.

- Self lensing. For a configuration such that the lenses and the sources belong to the target galaxy, we appreciate an increase of the differential rate of microlensing events towards the LMC if we use a non-Gaussian VD to describe the motion of its stars in place of a Gaussian one (see Figure 1). This increase is reflected upon the estimate of the number of expected events which is of roughly $35 \%$ larger as respect the 1.2 events found in the Gaussian case.

- MACHOs. In the case in which the velocity distribution of the sources in the LMC is considered to have a gaussian form, the prediction for a Halo fully composed by MACHOs is a factor $\sim 5$ above the observed rates. The situation does not change in a noticeable way if 
we consider a non-Gaussian VD, since we have found that the number of events expected is practically equal to the previous case. The results remain valid for both the DT and the universal VD. The great difference between the velocity dispersion of the two populations, $\sigma=20.2 \mathrm{~km} / \mathrm{s}$ for the LMC stars and $\sigma=155 \mathrm{~km} / \mathrm{s}$ for the MACHOs, practically neutralizes any possible variation due to the different shape of the VD of the sources.

The maximum-likelihood analysis gives values for $m_{\mathrm{ML}}$ and $f_{\mathrm{ML}}$ quite similar between the Gaussian and the non-Gaussian case. We conclude that the error on the estimate of the most probable value for the MACHO mass as well as for the Galactic halo fraction in form of MACHOs, calculated with a Gaussian VD for the LMC sources, is roughly of the order of $1-2 \%$. This fact means that in the study of the MW halo composition by microlensing, a Gaussian profile is a reasonable approximation for the velocity distribution of a system of source stars even if they are not spherically distributed.

On the other hand, in the study of self lensing, the Gaussian does not represent a good approximation to describe the kinematics of a non-spherically distributed stellar population, similar to the disk or the bar of the LMC. To ensure accurate microlensing prediction, it is thus necessary to replace the Gaussian VD by a more physically motivated one which takes into account the real spatial distribution of the source stars.

\section{References}

[1] J. Binney \& M. Merrifield, Galactic Astronomy, Princeton Univ. Press, Princeton NJ 1998.

[2] C. Alcock, R.A. Allsman, D.R. Alves et al., Astroph. Journal 5422812000.

[3] S. Calchi Novati, S. Paulin-Henriksson, J. An et al. Astron. \& Astroph. 4439112005.

[4] P. Tisserand, L. Le Guillou, C. Afonso et al., Astron. \& Astroph. 4693872007.

[5] J.T.A. de Jong, K. Kuijken, A.P.S. Crots et al., Astron. \& Astroph. 4174612004.

[6] J. Binney \& S. Tremaine, Galactic Dynamics, Princeton Univ. Press, Princeton NJ 1987.

[7] O. Iguchi, Y. Sota, T. Tatekawa et al., Phys. Rev. E71 0161022005.

[8] S.H. Hansen, B. Moore, M. Zemp \& J. Stadel, J. Cosmology Astropart. Phys. 010142006.

[9] D.P. Bennett, Astroph. Journal 6339062005.

[10] Ph. Jetzer, L. Mancini \& G. Scarpetta, Astron. \& Astroph. 3931292002.

[11] L. Mancini, S. Calchi-Novati, Ph. Jetzer \& G. Scarpetta, Astron. \& Astroph. 427612004.

[12] R.J. Assef, A. Gould, C. Afonso et al., Astroph. Journal 6499542006.

[13] S. Calchi Novati, F. De Luca, Ph. Jetzer \& G. Scarpetta, Astron. \& Astroph. 4594072006.

[14] C. Alcock, R.A. Allsman, D.R. Alves et al., Nature 4146172001.

[15] N. Kallivayalil, B.M. Patten, M. Marengo et al., Astroph. Journal 652 L97 2006.

[16] C. Alcock, R.A. Allsman, D.R. Alves et al., Astroph. Journal 5412702000.

[17] R.P. van der Marel, D.R. Alves, E. Hardy \& N.B. Suntzeff, Astron. Journal 12426392002.

[18] A. de Rújula, G.F. Giudice, S. Mollerach \& E. Roulet, Mon. Not. R. Astron. Soc. 2755451995.

[19] G. Chabrier, Astroph. Journal 55412742001. 